\title{
Statistical analysis of Sunspot Area and their Heliospheric Effect for the Period 1986 -2016
}

\author{
Prithvi Raj Singh, ${ }^{a, *}$ S. L. Agrawal, ${ }^{b}$ C. M. Tiwari ${ }^{b}$ and Abhay Kumar Singh $^{a}$ \\ ${ }^{a}$ Department of Physics, Banaras Hindu University, \\ Varanasi-221005, India \\ ${ }^{b}$ Department of Physics, A.P.S. University, \\ Rewa-486003, India \\ E-mail: prithvisingh77@gmail.com
}

\begin{abstract}
Sunspot area varies over the Sun's disk and is to be heliospheric behaviour during the descending phase of solar cycle 22 to 24 . Galactic Cosmic rays encounter an outward-moving solar wind with cyclic magnetic field fluctuation and turbulence. This causes convection and diffusion in the heliosphere. We have observed that the galactic cosmic rays recoveries are much faster than the solar parameter (sunspot area) with negative time lag during the descending phase of solar cycles 22 to 24. Statistical analysis of absolute asymmetry (A) of sunspot area is carried out for quasi-biennial (QBO) period is $\sim 1.94$ years with high amplitude during 2001. The significant Rieger-type periods ( $\sim 124$ to $\sim 175$ days) of absolute asymmetry (A) of the sunspot area have been investigated using Morlet Wavelet Techniques (MWT) for combined solar cycles 22 - 24.
\end{abstract}

$37^{\text {th }}$ International Cosmic Ray Conference (ICRC 2021)

July 12th - 23rd, 2021

Online - Berlin, Germany

\footnotetext{
${ }^{*}$ Presenter
} 
Keywords - Solar activity, Sunspot Area of the Sun, Galactic Cosmic rays

\section{Introduction}

The northern $(\mathrm{N})$ and southern $(\mathrm{S})$ hemispheres of asymmetry can be caused by the phase shift between magnetic activity in the hemispheres and the appearance of sunspots on the solar surface of the Sun (Singh et al., 2021; Singh et al., 2019a; Chowdhury et al., 2013 and references therein). Charbonneau (2005) observed periodic variations of solar activity indices exhibit different timescales ranging from minutes to centuries. The mid-term periodicities exhibit different timescales between $\sim 27$ days and $\sim 11$ years periods (Chowdhury et al., 2013, Singh et al., 2018, Singh et al., 2019b, and references therein). The various solar activity indices of North (N) - South (S) asymmetry data also exhibit different types of periods (Carbonell et al. 1993, Oliver \& Ballester 1994, Chowdhury et al., 2013; Singh et al., 2021; Singh et al., 2019a). They are also observed Rieger-type periodicities ( $\sim 120$ to $\sim 190$ days) and quasi-biennial oscillations $\sim 1.30$ years.

Modulation of the GCRs is a complex phenomenon that occurs throughout the heliosphere and depends on several factors: convection due to solar wind; curvature and slope of the interplanetary magnetic field; diffusion and adiabatic energy. The time series of sunspot area (SSA) is inversely proportional to Galactic Cosmic rays (GCRs) at observed the earth. It has been previously reported that the 11-year modulation of the GCRs intensity shows some time lag behind the solar activity indices (Singh et al., 2018, Chowdhury and Kudela, 2018 and references therein). Each of the time series moves backward or forward by one month or solar rotation (27-days) concerning the GCRs flux recorded at different neutron monitor stations (Chowdhury and Kudela, 2018).

In this study, we have analyzed the heliospheric behavior of the sunspot area during descending phase of cycles 22 to 24 (1986-2019). The analysis is carried out for the QBO period variations of absolute asymmetry data of sunspot area for the period 1986 - 2016. It is also observed mid-term periodicities including Reiger-type periodicities.

\section{Methods and Data Collection}

We considered monthly data of sunspot area for northern and southern hemispheres of the Sun for period January 1986 to September 2016. The monthly data of sunspot area of Northern (N) and Southern hemispheres (S) of the Sun have been taken from (http://solarscience.msfc.nasa.gov/greenwch.shtml). Total sunspot area on a monthly based for period January 1986 to September 2016 is taken from (https://solarscience. msfc.nasa.gov/greenwch/sunspotarea.txt) as well as for September 2016 to December 2019 taken from (https://www.spaceacademy.net.au). The pressure corrected monthly averaged data of GCRs measured by Moscow NM station $\left(2.42 \mathrm{GV}, 55.47^{\circ} \mathrm{N}, 37.32^{\circ} \mathrm{E}\right)$ taken from (http://cr0.izmiran.rssi.ru/mosc/main.htm).

To investigate the time-lag with sunspot area for different time variations and different solar magnetic field polarities during solar cycles 22-24. We have studied mid-term periodicities in the absolute asymmetry (A) by using the Morlet wavelet power spectrum method. The Morlet wavelet power spectrum is localized as a concept of Fourier analysis (Torrence and Compo, 1998). In the wavelet power spectrum, the cone of influence (COI) is defined as edge effects that occur in the beginning and end of time series. In Wavelet Analysis the dimensionless frequency $\omega_{o}=12$. It provides reasonable spatial and temporal resolutions (Torrence and Compo 1998).

The statistical analysis of the absolute asymmetry (A) has been calculated as follows: 


$$
\text { Absolute asymmetry }(\mathrm{A})=(\mathrm{N}-\mathrm{S}) \text {, }
$$

where $\mathrm{N}$ and $\mathrm{S}$ are values of the northern and southern hemispheres of the sunspot area respectively.

\section{Results and Discussions}

\subsection{Variations of Northern $(\mathrm{N})$ and Southern (S) hemisphere of Sunspot area}

We observed amplitude variation of sunspot areas such as northern and southern hemispheres of the Sun and it shows rise gradually and fall patterns during the study period shown in Figure 1. The amplitude of the sunspot area shows a shift of solar activity from northernto southern hemisphere for the period 1990, 2001, and 2012. The northern hemisphere is dominated at the very beginning of cycle while southern hemisphere dominates in decline phase of the cycle during the study period.

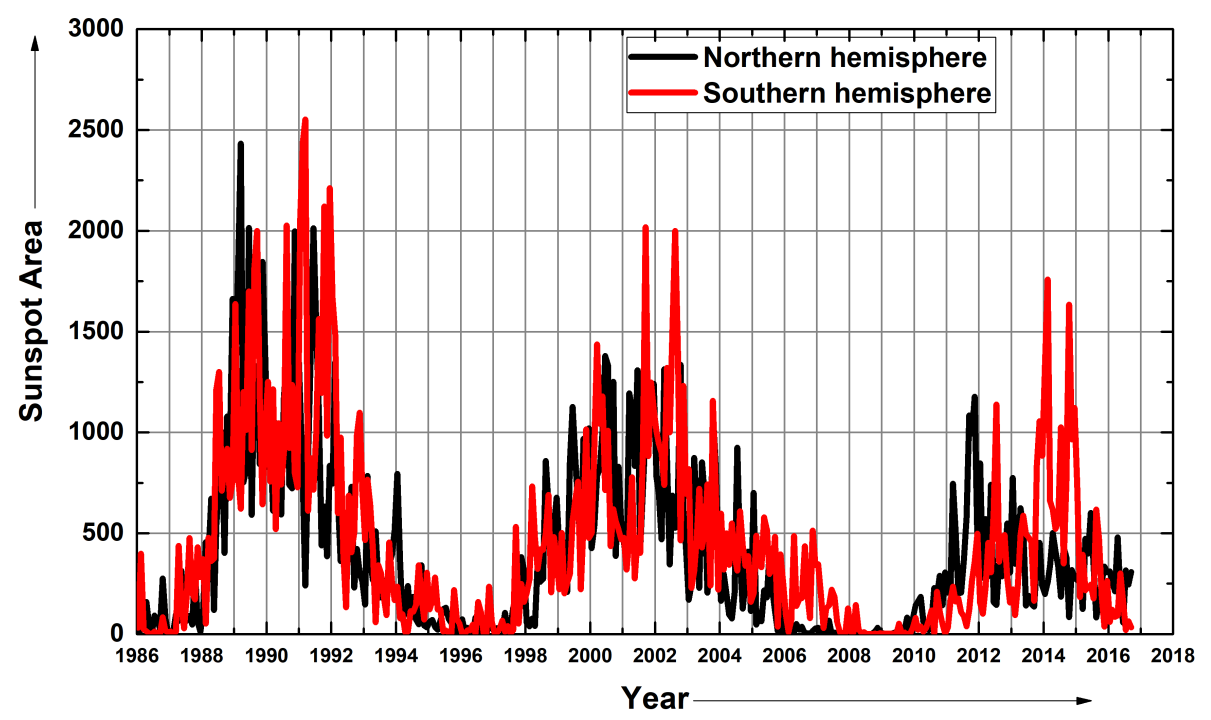

Figure 1: The monthly variation of the northern hemisphere (black line) and southern hemisphere (red line) of the Sunspot Areas for the period $1986-2016$.

Solar activity has independent in both hemispheres and, it is controlled by the solar rotation (magnetic polarity) of each hemisphere (Chowdhury et al., 2013; Singh et al., 2021; Singh et al., 2019a). If the direction of the field is outward from the Sun in northern hemisphere, then the polarity is positive $(\mathrm{A}>0)$ and the opposite occurs when the polarity is negative $(\mathrm{A}<0)$. The above polarity phenomenon is based on two components, namely: diffusion and convection are independent of polarity of the solar magnetic field and change only with magnetic force of solar activity.

The value of yearly based Absolute Asymmetry (A) of Sunspot Area (SSA) is shown in Figure 2 for period 1986 - 2016. The absolute asymmetry (A) method indicates both types of hemispheres are present in an asymmetry of sunspot area. The two hemispheres are asymmetrical between the years 1989 to 1991 , 1999 to 2002, and 2011 to 2014 with a high diffusivity forced (magnetic field) present in that year. Solar cycle 23 shows pronounced double peaks in case of absolute asymmetry of sunspot area is connected with solar activity maximum in the photosphere. Our Absolute Asymmetry (A) techniques revealed the presence of phase shift between magnetic activities in the hemispheres on solar surface of the Sun. The differential 
rotation and meridional circulation in each hemisphere are related to the solar magnetic activity (Chowdhury et al., 2013). It means the change of dominant activity of northern maximum to southern maximum is connected with each other.

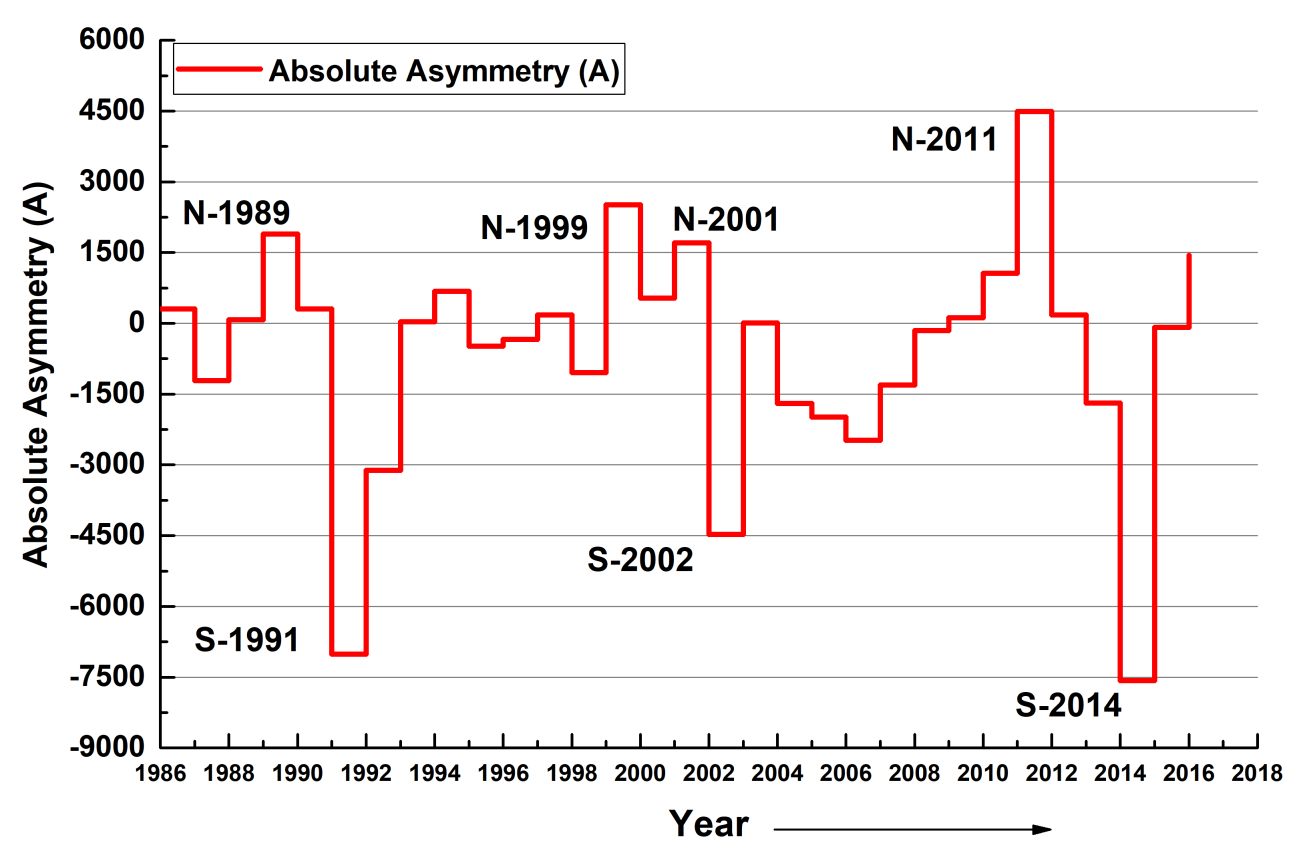

Figure 2: . The yearly variation of Absolute Asymmetry (A) of Sunspot Area for period 1986-2016.

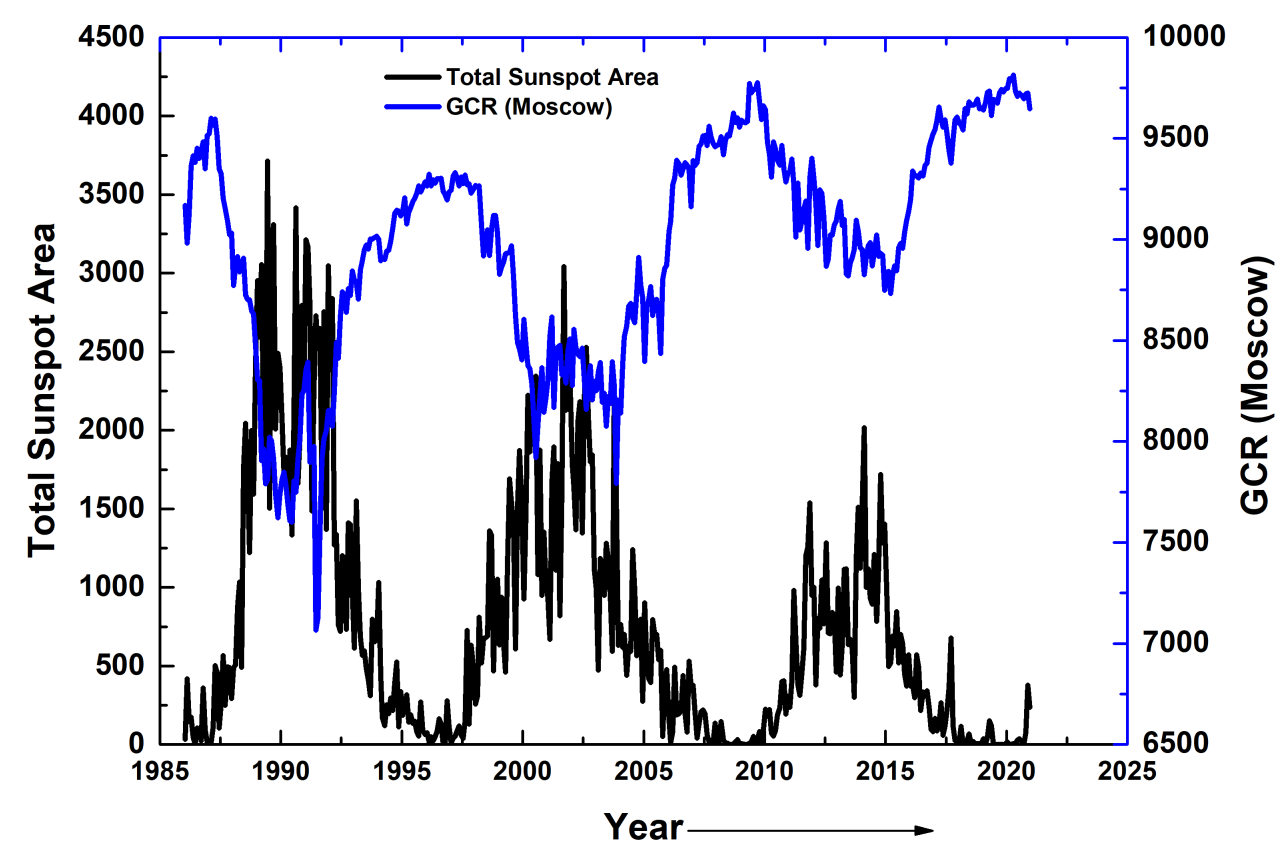

Figure 3: The monthly variation of the GCRs (Moscow) blue line and total sunspot area is a black line for the period 1986-2016. 
Figure 3 represents monthly averages of the Galactic Cosmic Rays (Moscow) and total sunspot area and their behaviour of flux variations during solar cycles 22-24 (1986 - 2020). GCR intensity gradually increases from the beginning of 1993 and 2006 and gradually decreases from the beginning of 1988 and 2010 but the trend is different for 2010 to 2014. The total sunspot area shows two prominent peaks; the second peak is larger than the first one. GCR intensity and solar activity (sunspot area) are strongly anti-correlated during the study period as shown in Figure 3.

\subsection{Heliospheric Effect on Sunspot area}

Figures 4, represent the time-lag between GCRs intensity and total sunspot area during the epoch of solar cycle 22-24 (descending phase) respectively, with their statistical errors. The correlation plot is nearly flat; we have considered the first and the second highest cross-correlation values and the corresponding time lag. From Figure 4 the change of phase and time lag is depending on the rate of change of GCRs intensity with the change in solar activity during solar cycles 22-24 (descending phase).

In the case of even-numbered solar cycles (22 and 24), the recovery rate of the GCRs is much faster than odd-numbered cycles (23) i.e. charged particles move through the polar region is smaller than odd-numbered solar cycles. Tables 1 , represent the values of the correlation coefficient and time lag between GCRs and sunspot area (SSA) during solar cycle 22-24 (descending phase). The average normalized correlation coefficient (with a time lag) between SSA and GCRs is 0.67. Thus sunspot area is highly correlated with the GCR intensity during the descending phase of cycle 23 see Table 1 . In this study, we observed the time lag between GCRs (Moscow NM station) and the sunspot area during the descending phase of solar cycles 23 is $\sim 12$ months. But during cycles 22 and 24 are similar time lag $\sim 4$ months. It means in the even cycle (22 and 24) for descending phase is minimum as compared to odd cycle 23.

On the above result, we concluded that the polarity of the solar magnetic field, positive polarity $(\mathrm{A}>0)$ is by magnetic field direction outward from the Sun, and the opposite is at $\mathrm{A}<0$. Our investigation exhibits a negative time lag between GCRs and SSA during the descending phase of cycles 22-24, which is consistent with the earlier results (Usoskin et al. 2001, Chowdhury et al. 2013, Chowdhury and Kudela, 2018). Some previous studies indicated that the time lag between GCRs and SSA is relatively small in even-numbered cycles in comparison with the odd-numbered cycles (Chowdhury and Kudela, 2018 and references therein).

Table 1: Normalized Cross-correlation coefficients (CC) between GCRs intensity (Moscow) and the sunspot area (SSA) with a corresponding time lag (L) during solar cycle 22-24 (descending phase).

\begin{tabular}{lll}
\hline Sunspot Area (SSA) & $\begin{array}{l}\text { Normalized CC (with 95\% signif- } \\
\text { icance level) }\end{array}$ & L (Months) \\
\hline Cycle22 (descending phase) & $0.665-0.657$ & $-5-0$ \\
Cycle23 (descending phase) & $0.683-0.675$ & $-12-0$ \\
Cycle24 (descending phase) & $0.664-0.656$ & $-4-0$ \\
\hline
\end{tabular}

\subsection{Periodicity of Absolute Asymmetry of Sunspot Area}

We observed mid-term periodicities as Reiger type, intermediate-term,quasi-annual and quasi-biennial period in the absolute asymmetry (A) of the sunspot area by Morlet wavelet power spectrum method during combined solar cycles 22 to 24 (1986 - 2016). We have found significant mid-term periodicities in absolute asymmetry (A) of the sunspot area: Reiger type $\sim 124$ days, $\sim 147$ days, $\sim 175$ days, intermediate-term $\sim 208$ days, 247 days, and quasi-annual period 350 days, 

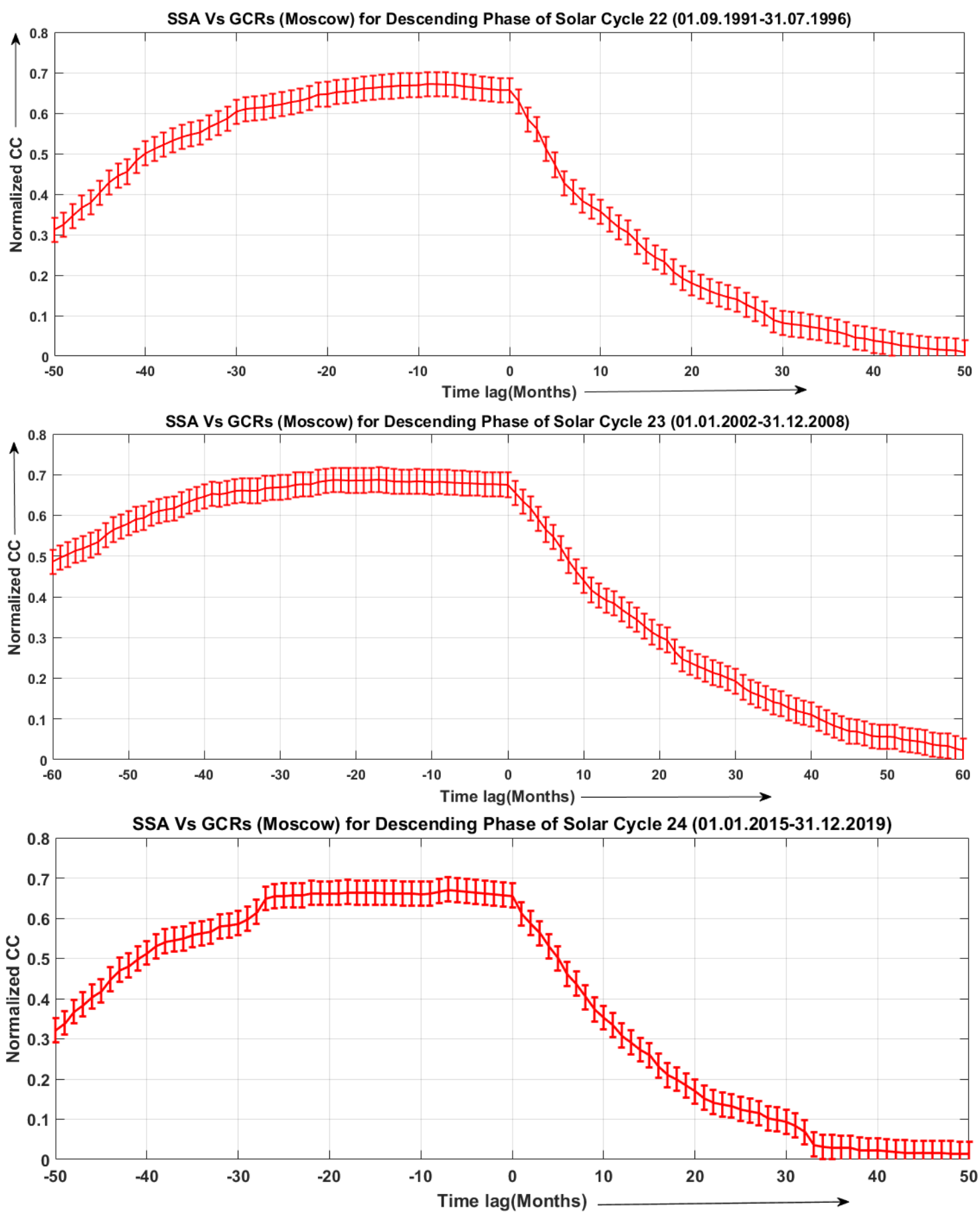

Figure 4: Cross-correlation coefficients and time-lag between GCRs with SSA for the descending phase of solar cycles 22, 23, and 24.

and quasi-biennial period $\sim 1.16$ years, $\sim 1.38$ years, $\sim 1.64$ years, $\sim 1.95$ years, $\sim 2.31$ years, $\sim 2.75$ years, $\sim 3.27$ years, and $\sim 3.89$ years.

Our result shows a period of absolute asymmetry of sunspot area is the rotational rate at the base of the 
solar convection zone is $\sim 1.38$ years during combined solar cycles $22-24$, whereas Chowdhury et al. (2013) observed $\sim 1.40$ years based on daily based data (normalized asymmetry value) during solar cycle 23 . The amplitude profile for asymmetry of sunspot area is representing the mid-term periodicity such as (Rieger type, quasi-period quasi-biennial period and quasi-annual period) show power with yellow colour in Figure 5.

From wavelet analysis, it has been detected that the nature and variation of the QBO are similar and present in all solar cycles in the SSA under the study period. The QBO, especially the variation $\sim 1.94$ years, is assumed as one of the fundamental variations of solar activities and it is probably intrinsic properties of the Sun connected to the dynamo mechanism in Figure 6. The QBO period for cycle 23 is largely shifted in the negative direction as compared to cycles 22 and 24. The result of a QBO period is supporting the $\mathrm{S}$-hemisphere dominance over the N-hemisphere during the study period.
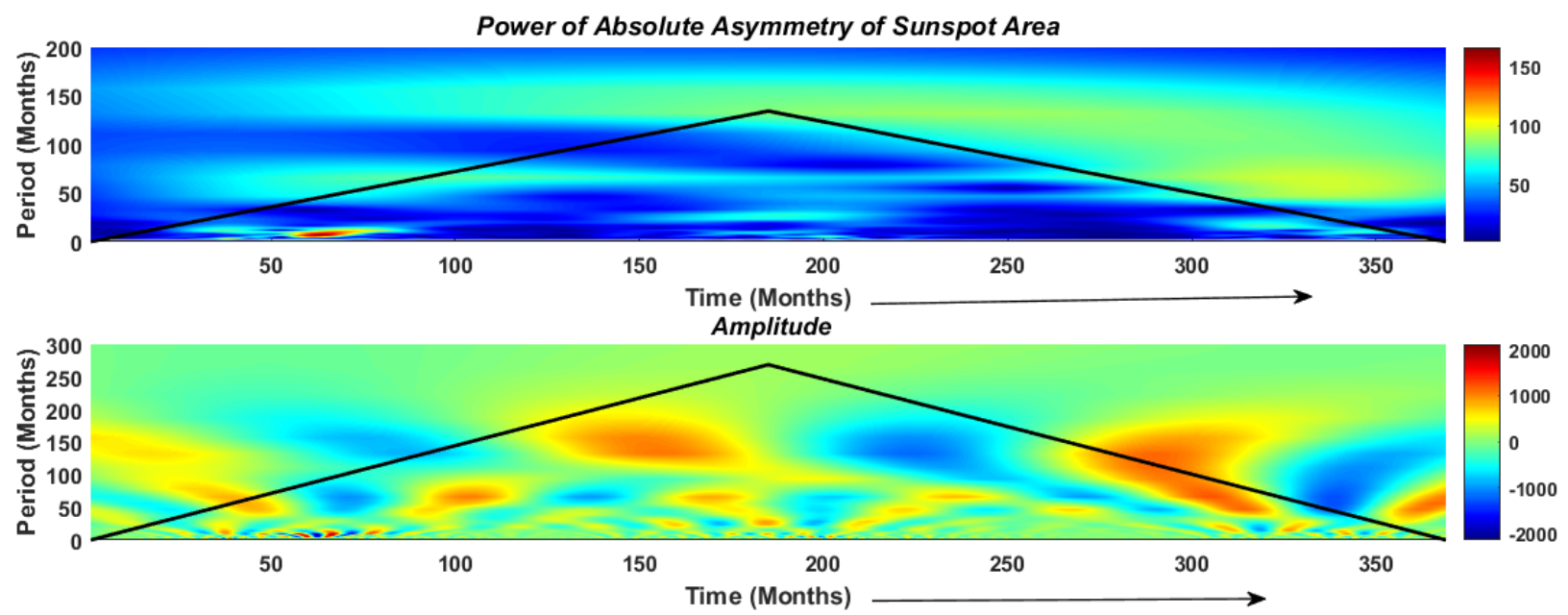

Figure 5: Periodicity of absolute asymmetry of Sunspot Area by Morlet Wavelet Power Spectrum Methodduring combined solar cycles 22 -24 (1986-2016).

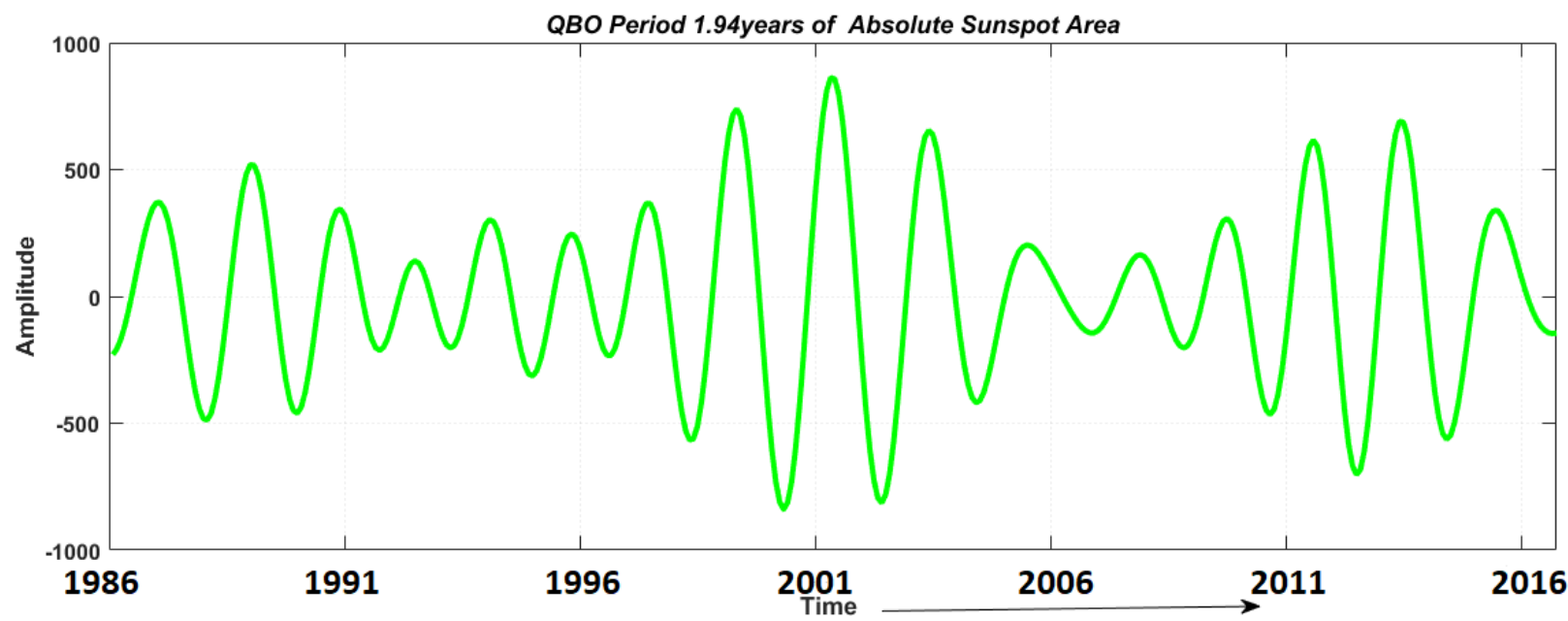

Figure 6: QBO Period of absolute asymmetry of Sunspot Area by Morlet Wavelet Power Spectrum Method during combined solar cycles 22 -24 (1986 - 2016). 


\section{Conclusion}

The amplitudes of northern and southern hemispheres showed decreased magnetic solar activity during cycles 22 to 24 . It was observed that cycle 24 is magnetically weak. The QBO period shows the change in phase and behavior of solar activity from one hemisphere to the other. QBO periods are evidence of a strong asymmetry in both sides of the solar equator. It is the highest power that appears around the period 2011.

The significant period $\sim 1.38$ years of absolute asymmetry of sunspot area is the rotational rate at the base of the solar convection zone. The significant periodicity of absolute asymmetry of sunspot area for the quasi-biennial period varies $1.16-3.89$ years. The period $\sim 0.97$ years ( $\sim 350$ days) of absolute asymmetry of sunspot area is prominent during the study period.

In this study, we examined the highest time lag between GCRs (Moscow NM station) and sunspot area during the descending phase of solar cycles 23 with $\sim 12$ months with maximum cross-correlation of $\sim 0.68$. This indicates that the recovery rate of the GCRs' during the descending phase of solar cycles 22 and 24 is much faster than the solar cycles 23.

\section{References}

[1] Carbonell, M., Oliver, R., Ballester, J. L. 1993, Asstrophy. and Astro., 274, 497.

[2] Charbonneau, P., 2005, LRSP, 2, 1rsp-2005-2.

[3] Chowdhury, P., and Kudela K., 2018, Astrophys Space Sci, 363,250.

[4] Chowdhury, P., Choudhary, D.P., Gosain, S., 2013, ApJ, 768, 188.

[5] Oliver, R., and Ballester, J.L., 1994, Sol. Phys., 152, 2, 481.

[6] Singh, P. R., Saxena, A.K. Tiwari, C.M., 2018, Journal Astrophys. Astro., 39, 20.

[7] Singh, P. R., Tiwari, C. M., Saxena, A. K., Agrawal, S. L., Mishra, A. P., 2019a. Astrophys Space Sci, 364(4), 59.

[8] Singh, P. R., Tiwari, C.M., Agrawal, S.L., Pant Tarun Kumar, 2019b, Sol. Phys., 294, 218.

[9] Singh, P. R., Farid, AI. S., Pant, T. K., Singh A. K., 2021. Res. Astro. Astrophys, 21(4), 106.

[10] Torrence, C., and Compo, G. P. 1998, BAMS, 79, 61.

[11] Usoskin, I.G., Mursula, K., Kananen, H., Kovaltsov, G.A., 2001, Adv. Space Res. 27(3), 571. 